\title{
Microwave dielectric spectroscopy for single cell irreversible electroporation monitoring
}

\author{
Amar TAMRA $^{1,2}$, Marie DEBURGHGRAEVE ${ }^{1}$, David DUBUC ${ }^{1}$, Marie-Pierre ROLS ${ }^{2}$, Katia GRENIER ${ }^{1}$ \\ ${ }^{1}$ LAAS-CNRS and University of Toulouse, ${ }^{2}$ IPBS-CNRS, Toulouse, France
}

\begin{abstract}
This paper demonstrates the efficient use of microwave dielectric spectroscopy for single cell irreversible electroporation monitoring. The experimental results point out a high correlation $\left(R^{2}\right.$ higher than 0.94$)$ with biological gold standard technique based on flow cytometry, whereas microwave approach features several key advantages: marker-less, contactless and in-liquid cell(s) monitoring. The developed microwave and microfluidic-based biosensor reveals an increase in the capacitance and the conductance of single cells under investigation subjected to irreversible electroporation reflecting an increase in cell damages, which is in great correlation with results routinely obtained in biology. More interestingly, the post electroporation damages of cell have been experimentally quantified over time, which makes the microwave dielectric spectroscopy a technique of interest for cell's electroporation researches.

Index Terms - Dielectric spectroscopy, Electroporation, Biosensor, Single cell analysis, microwave
\end{abstract}

\section{INTRODUCTION}

Accessing the intracellular domain of viable cells has shown a wide interest in the area of biomedical applications. This access can be mediated by several means, such as electroporation. It enables the insertion of proteins into cell membrane, cell fusion, eradication of bacteria, the introduction of small and large molecules into the cell, as well as tumor ablation [1]. Electroporation EP (also known as electropermeabilization), is a physical method that consists in applying well controlled electric pulses (in amplitude and duration) [2], in order to transiently permeabilize the plasma membrane of cells and thus allowing the entry and exit of molecules of interest [3]. In some cases, this approach can cause permanent cell damages and results in cell death: irreversible electroporation (IRE) [4]. When electric pulses are applied to the cell, the electric potential $\Psi$ of the cell membrane is modified: when the sum of the native transmembrane potential $\Delta \Psi 0$ and the induced transmembrane potential $\Delta \Psi \mathrm{i}$ exceeds a threshold value $(-200 \mathrm{mV})$, the permeabilization occurs [5].

Despite progress in this field, enlarging our knowledge about the mechanism of cell electroporation and kinetics associated with electrochemotherapy remains a pressing need. The assessment of cell permeability and viability after exposure to electroporation is basically done by using conventional microscopy with fluorescent markers, such as calcein AM and propidium iodide (PI), and conventional viability tests such as MTT (Tetrazolium Reduction Assay): measuring the mitochondrial activity of cells. These methods are time consuming, require important volumes of materials, and more importantly are invasive to the cells.

Aside from these techniques, new bio-physical methods, such as Atomic Force Microscopy (AFM) and electrical spectroscopy, have consequently been developed to enrich the cell's physical mechanisms description. The measurement of dielectric properties of biological samples is undeniably expected as of interest to monitor both membrane and intracellular content of the biological cells [6]. When using sub-GHz Electrical Impedance Spectroscopy (EIS), the membrane state (permeabilization) is indeed revealed [7]. Operating at microwaves (beyond few $\mathrm{GHz}$ ) becomes then particularly interesting, because (1) the cell's membrane becomes transparent [8], and consequently (2) the electromagnetic fields penetrate deeply into the cell and interact directly with the intracellular content.

Here we report our work on the exploitation of our microwave and microfluidic-based biosensor, operating at the single cell level, to monitor the intracellular effects of irreversible electroporation on cells under investigation. We first describe the micro-device architecture combining microfluidic and electrical functionalities. A second part deals with our results on the microwave monitoring of cells submitted to irreversible electroporation; their strong correlation with data obtained with flow cytometry clearly points out the efficacy of our technique in depicting the kinetic effects of cell's electroporation.

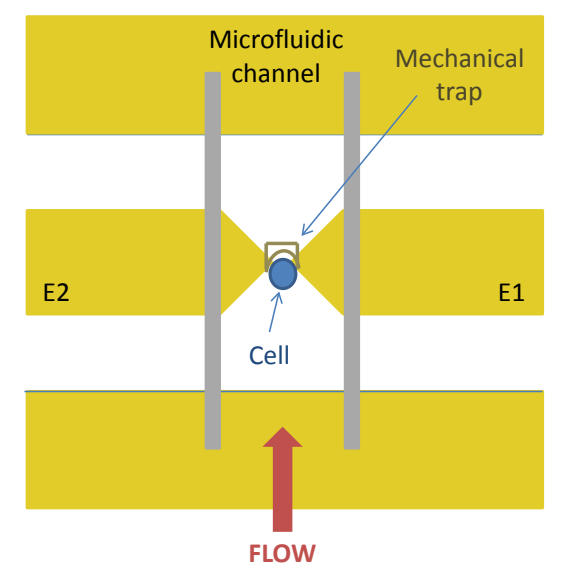

Fig.1: Schematic top view of the biosensor composed of a coplanar waveguide with a microfluidic channel placed on top; the cell is blocked due to the mechanical trap. 


\section{MATERIALS AND METHODS}

This work is based on a micro-biosensor developed by Chen et al. [9], which takes benefits from the convergence between (1) microfluidic functionalities in charge of the processing of biological material (pure cultured medium or medium with cytotoxic agents or cells suspension) and (2) microwave sensor for marker-less, contact-less and in-liquid single cell monitoring. The micro device used to analyze biological cells is presented in (Fig.1): It is composed of a coplanar waveguide with a capacitive gap in the center. On top of it is placed a microfluidic channel, with a mechanical trap in its center which will help capturing and probing one single cell. The cell's analysis is based on the interaction between electromagnetic fields and the biological sample, which reflects the dielectric properties of cells and makes it possible to assess its state in real time.

In this study, we use THP1 cell line (ATCC\#TIB-202): a cell line derived from the peripheral blood of an acute monocytic leukemia patient. The traditional cell culture medium is the Roswell Park Memorial Institute one (RPMI) with $10 \%$ of Fetal Bovine Serum (FBS). Electric pulses are delivered to the cells using a Betatech pulse generator with two stainless steel electrodes with a distance of $4 \mathrm{~mm}$. The used electrical parameters are 8 pulses of $1.5 \mathrm{kV} / \mathrm{cm}$ at pulse duration of $100 \mu \mathrm{s}$ with a frequency of $1 \mathrm{~Hz}$. The choice of these parameters was made based on the permeability of cells after EP (Fig.2) assessed by PI intake: propidium iodide is a small molecule that becomes fluorescent when bound to cellular RNA or DNA. Hence, it only marks cells with permanently or transiently compromised membrane integrity [10].

We wanted to evaluate the effect of irreversible electroporation on THP1 cells by studying their response through time. The experimental protocol is composed of 2 major steps. First, cells were pulsed with the generator and reincubated at $37^{\circ} \mathrm{C}$ and $5 \% \mathrm{CO} 2$ for 3, 5 and 7 hours after EP. At the end of the incubation time, cells were injected in the RF microdevice or in the flow cytometer in order to compare both results.

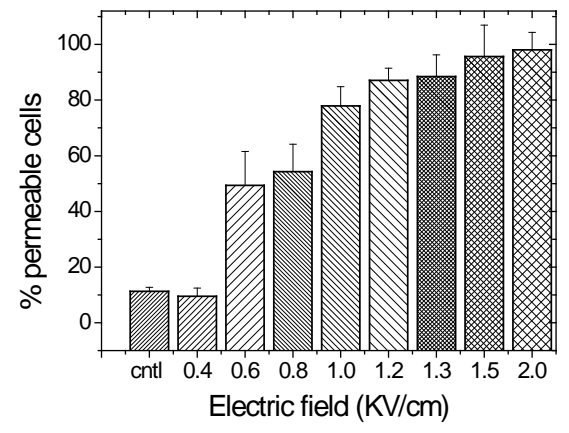

Fig.2: Evaluation of THP1 permeability post EP by PI intake.

\section{MICROWAVE MEASUREMENTS}

\section{A. Irreversible electroporation identification}

The microwave measurements are performed from $40 \mathrm{MHz}$ to $40 \mathrm{GHz}$. The culture medium is first measured alone and considered as our reference medium. When the cells are injected into the microfluidic channel, one cell is captured due to the mechanical trap, directly on top of the sensing zone. Two readouts [9], the effective capacitance $C$ and conductance $G$ of the sensing volume, are extracted with a specific procedure [11], and compared to the reference medium by calculating contrasts as indicated below:

$$
\Delta \mathrm{C}=\mathrm{C}_{\text {cell }}-\mathrm{C}_{\text {reference medium }}, \Delta \mathrm{G}=\mathrm{G}_{\text {cell }}-\mathrm{G}_{\text {reference medium }}
$$

Fig.3 shows the spectrums of contrast of capacitance and conductance obtained several hours post EP. As shown in Fig.3, both contrasts decrease (in absolute values) after exposure to electric pulses reflecting cell death due to irreversible electroporation. This may be explained by the fact that permeable cells have greater exchange with the reference medium, thus the contrast between these two samples is less important. This study allowed us to establish a threshold value between alive and affected cells at the middle of the measured dynamic: $\Delta \mathrm{C}_{\mathrm{thr}}=-0.38 \mathrm{fF}$ at $5 \mathrm{GHz}$ and $\Delta \mathrm{G}_{\mathrm{thr}}=-35 \mu \mathrm{S}$ at $30 \mathrm{GHz}$. The frequencies on both parameters have been selected to obtain the maximum dynamic as suggested in Fig.3.
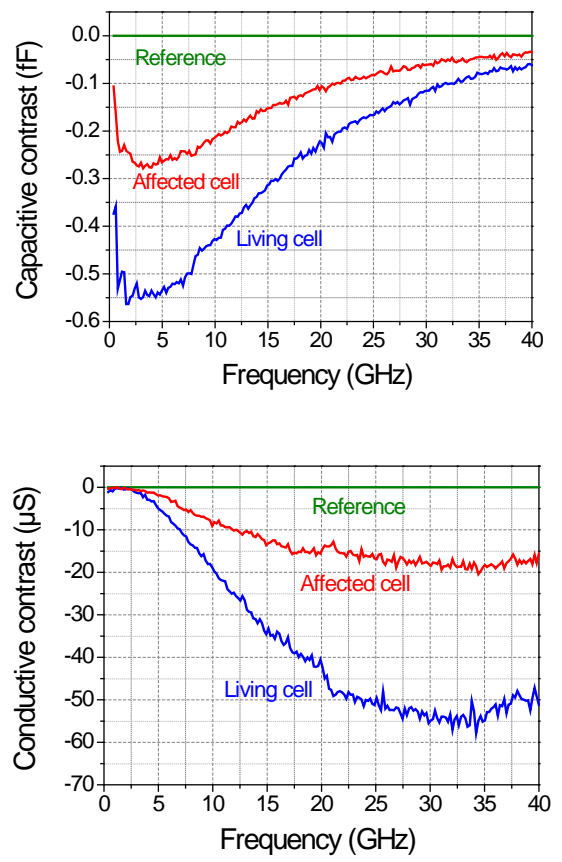

Fig. 3: Capacitive and conductive contrasts of a living cell and an affected cell several hours after electroporation. 


\section{B. Monitoring of irreversible electroporation kinetics}

After depicting the different electric signatures of a living and an irreversibly electroporated cell, the next step was to demonstrate the ability of such a technique to monitor the kinetic effect of the IRE. As explained in the materials and methods, the cells were electroporated and studied 3,5 or 7 hours after EP. At least 10 measurements have been performed for each condition. Once the measurements were done, we proceeded to a cell sorting and a classification based on the thresholds established earlier.

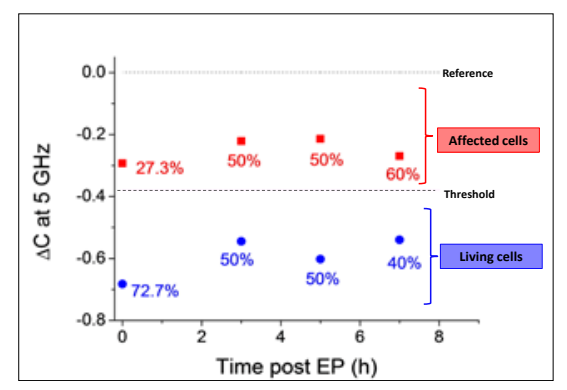

Fig.4: Mean values of the contrast of capacitance obtained different hours after EP. Percentages of viable and affected cells are presented.

Fig. 4 presents the mean values of the contrasts obtained after classification as well as the different percentages of cells: viable or affected by the IRE. Compared to the non-treated cells, which feature a viability level (number of viable cells divided by the total cells number) around 73\%, IRE induce a decrease of the viability down to $50 \%$ after 3 and 5 h post IRE and down to $40 \%$ beyond 7 hours after the electrical stress. As one can notice, the mean value of contrasts (same results are obtained on the conductance) is constant after IRE. Only the number of cells in each state varies over time.

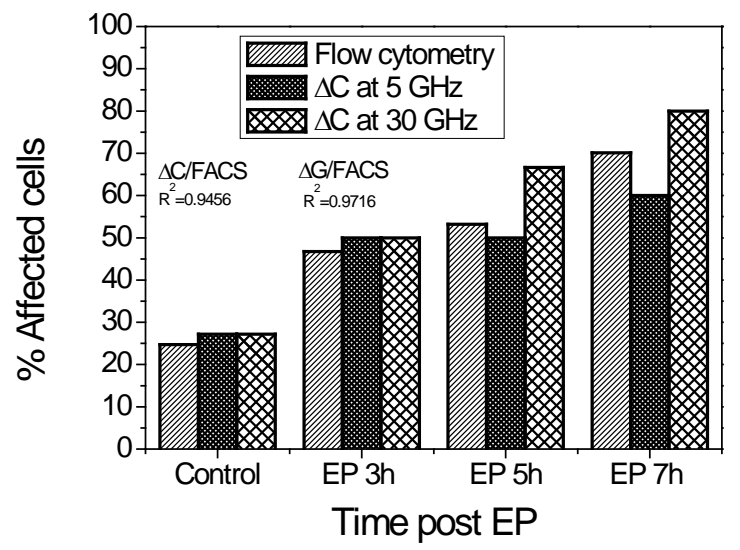

Fig.5: Correlation between results obtained with microwave measurements (contrasts of capacitance and conductance) and Flow cytometry.

\section{DISCUSSION}

The potential of the label-free microwave technique to detect cell death after IRE was evaluated by comparing the results obtained with the well-established flow cytometry technique using PI dye to reveal cell death. The bioexperiments described in part II were conducted with both microwave and flow cytometry techniques.

Fig.5 represents the percentage of dead cells post EP obtained with flow cytometry and microwave measurements (based on both contrasts of capacitance and conductance). A statistical analysis was performed to correlate experiments and it points out strong correlation coefficient $\mathrm{R}^{2}$ between $\Delta \mathrm{C} /$ flow cytometry $\left(\mathrm{R}^{2}=0.94\right)$ and $\Delta \mathrm{G} /$ flow cytometry $\left(\mathrm{R}^{2}=0.97\right)$. These correlations clearly reveal the efficacy and robustness of our technique in depicting the kinetic effects of cell's electroporation.

\section{CONCLUSION}

In this paper, we experimentally demonstrate that the microwave dielectric spectroscopy of single cells significantly reveals the cell's damages after irreversible electroporation and is able to monitor these damages over time.

Experimental investigations driven by biologists indeed point out that microwave readouts feature a high correlation ( $\mathrm{R}^{2}$ of 0.94 on $\Delta \mathrm{C}$ and 0.97 on $\Delta \mathrm{G}$ ) with biological gold standard technique based on flow cytometry.

These results convince biologists that microwave dielectric spectroscopy may enrich the technique's arsenal for cell's electroporation researches. The technique could contribute to provide more insights on the effect of electroporation and electrochemotherapy on cancer cells.

\section{REFERENCES}

[1] T. Kotnic et al, Electroporation-based applications in biotechnology. Trends in Biotechnology (2015); 33:480-488.

[2] J. Teissie, M. Golzio and M. P. Rols, Mechanisms of cell membrane electropermeabilization: a minireview of our present (lack of?) knowledge. Biochim Biophys Acta (2005); 1724(3): 270-280.

[3] E. Neumann, and K. Rosenheck, Permeability changes induced by electric impulses in vesicular membranes. J Membr Biol (1972); 10(3): 279-290.

[4] B. Rubinsky, G. Onik and P. Mikus, Irreversible electroporation: a new ablation modality--clinical implications. Technol Cancer Res Treat (2007); 6(1): 37-48.

[5] J. Teissie, and M. P. Rols, An experimental evaluation of the critical potential difference inducing cell membrane electropermeabilization. Biophys J (1993); 65(1): 409-413.

[6] D. Pavlidis, High Frequency Wideband Permittivity Measurements of Biological Substance Using Coplanar Waveguides and Application to Cell Suspensions, IEEE MTT-S, 2008, pp. 915- 918.

[7] D.A. Dean et al. Electrical Impedance Spectroscopy Study of Biological Tissue, Journal of electrostatics (2008); 66.3-4, pp. 165-177.

[8] C. Dalmay et al. Development of High Frequency Microfluidic Biosensors for Intracellular Analysis, EUROSENSORS (2014); Volume 87, pp. 54-57.

[9] T. Chen et al, Microwave biosensor dedicated to the dielectric spectroscopy of a single alive biological cell in its culture medium. IEEE IMS (2013); 978-1-4673-2141. 
[10] V.L.Sukhorukov et al, Surviving high-intensity field pulses: strategies for improving robustness and performance of electrotransfection and electrofusion. J.Membr.Biol.206 (2005)187-201.

[11] W. Chen et al, Microwave Dielectric Spectroscopy of a Single Biological Cell with Improved Sensitivity up to $40 \mathrm{GHz}$. IEEE MTT-S, IMS 2015; 17-22 May 2015. 